\title{
Génotypage des Virus du Papillome Humain (VPH/HPV) dans les lésions précancéreuses et cancéreuses du col utérin à Bamako (Mali)
}

\section{Genotyping of Human Papillomavirus (HPV) in precancerous and cancerous lesions of the cervix in Bamako}

\author{
Konaté $\mathrm{A}^{1}$; Sissoko S. $\mathrm{B}^{2}$; Coulibaly $\mathrm{B}^{2}$; Sow $\mathrm{A}^{2}$; Keïta $\mathrm{M}^{2}$; Maïga $\mathrm{R}^{2}$; Traoré C. $\mathrm{B}^{2}$; Kamaté $\mathrm{B}^{2}$; Bayo $\mathrm{S}^{3}$; Simporé $\mathrm{J}^{4}$.
}

\author{
1 : Centre de Santé Communautaire Universitaire (CSCom U) \\ de Ségué \\ 2 : Service d'Anatomie et Cytologie Pathologiques du C.H.U. du \\ Point $\mathrm{G}$; \\ 3 : Laboratoire d'Anatomie et Cytologie Pathologiques de la \\ polyclinique Pasteur-Bamako. \\ 4 : Centre de Recherche Biomoléculaire Pietro Annigoni de \\ Ouagadougou.
}

Correspondance : Dr Aboubakary KONATE, Centre de Santé Communautaire Universitaire (CSCom U) de Ségué, Bamako Mali ; Tel : (00223) 66562052 ; Email :

aboubakarykonate@gmail.com

\section{Résumé :}

Introduction: Le cancer du col de l'utérus est, dans $99 \%$ des cas, dû à un Virus Papillome Humain à Haut Risque (HR-HPV). Cette étude a été initiée pour déterminer les types d'HPV associés aux lésions précancéreuses et cancéreuses du col utérin à Bamako. Matériel de méthodes: Nous avons mené une étude transversale prospective, entre le 1er Janvier 2016 et le 30 Avril 2017. Les sites étaient d'une part le service d'Anatomie et Cytologie Pathologiques du Centre Hospitalier Universitaire (CHU) du Point-G (BamakoMali) où l'examen histologique des fragments de biopsies et des pièces opératoires, provenant des centres de dépistages, a été réalisé et d'autre part le Centre de Recherche Biomoléculaire Pietro Annigoni (CERBA/LABIOGENE) de Ouagadougou (Burkina Faso), pour le génotypage des HPV des échantillons recueillis. Avec la technique PCR multiplex en temps réel, nous avons recherché 14 génotypes du HR-HPV dans 192 prélèvements de Lésions Intra-Epithéliales de Haut Grade (LIEHG) et de cancers invasifs du col de l'utérus. Les données ont été analysées sur le logiciel SPSS 18.0. Résultats: Seulement 43,2\% (83/192) avaient un résultat positif. Le génotypage a révélé la présence de 13 génotypes HR-HPV, les plus fréquents étaient HPV18 (16,3\%), HPV45 (16,3\%). Les fréquences d'infections uniques et multiples étaient respectivement de 77,1 et $22,9 \%$. La majorité des femmes avec un HPV positif était des multipares avec 30,9\%. Le type histologique le plus représenté était les Néoplasies Intra-épithéliales modérées (CIN2) avec 42,2\%. Conclusion : L'approche moléculaire doit faire partir de l'arsenal des techniques utilisées dans le dépistage du cancer du col pour une meilleure prise en charge.

Mots clés: génotypage, virus papillome humain, col, utérus, cancer, Bamako.

\section{Abstract:}

Introduction: The human papilloma virus high risk (HRHPV) is responsible of $99 \%$ cases of cervix cancer. This study was initiated to determine the types of HPV associated with precancerous and cancerous lesions of the cervix in Bamako. Material de methods: We did cross sectional prospective study form January 2016 to April 2017. Study sites was in one hand the department of anatomy and cytology pathologies of university hospital of point $\mathrm{G}$ (Bamako, Mali) were the histological examination of the fragments of biopsies and operative parts, coming from the screening centers, was carried out and the other hand, the molecular biology research center "Pietro Annigoni (CERBA/LABIOGENE)" of Ouagadougou (Burkina Faso) were the molecular biologic study has been realized. We did the genotyping of human papilloma virus. We searched 14 genotypes of high risk of human papilloma virus (HR-HPV) with the PCR technic at real time in 192 cervix lesions. The data analyzed on the SPSS 18.0. Results: Only $43.2 \%$ (83/192 cases) have positive test. We obtained 13 genotypes HR-HPV and the most frequent was HPV18 (16.3\%), HPV45 (16.3\%). The unique and multiple infections was respectively 77.1 and $22.9 \%$. The woman HPV positive was multiparous in $30.9 \%$. Conclusion: The most common histological type was moderate intraepithelial neoplasia (CIN2) with 42.2\%. the molecular approach was indispensable in the arsenal technic of detection.

Keywords: genotyping, human papillomavirus, cervix, uterus, cancer, Bamako.

\section{Introduction :}

Le cancer du col utérin est une néoformation tissulaire due à une prolifération cellulaire excessive, anormale, anarchique, et autonome qui se développe aux dépens du col de l'utérus [1]. A l'échelle mondiale, le cancer du col de l'utérus est le 2ème cancer chez les femmes avec une fréquence de $15 \%$ après celui du sein [2,3]. Au Maghreb le cancer du sein a pris la 1ère place comme dans les pays occidentaux, le cancer du col utérin est devenu beaucoup moins fréquent avec une incidence de 2,4\% [4]. En Afrique subsaharienne francophone le cancer du col utérin est très fréquent avec une incidence de $15,5 \%$ [4]. Au Mali, il vient en première position, avec une fréquence de $26,6 \%$ et une incidence de 35,1 pour 100000 habitants [1].

Cependant la prévention de ce cancer devra encore reposer sur la détection précoce des lésions précancéreuses par les méthodes d'inspection visuelle ou par les frottis cervico-vaginale ou encore par les tests HPV. 
Par ailleurs les infections aux papillomavirus humains en particulier à ceux appartenant aux génotypes à haut risque oncogène (HPV16, 18, 31, 33, 35, 39, 45, 51, 52...) sont fortement associées à l'apparition des lésions précancéreuses et cancéreuses du col de l'utérus. La distribution et la fréquence de ces types oncogènes d'HPV diffèrent fortement selon les régions géographiques. Cette différence peut avoir un retentissement négatif sur l'efficacité des vaccins déployés, qui visent les deux types les plus fréquemment retrouvés (HPV 16 et 18) dans les lésions précancéreuses et cancéreuses du col [2]. Cette étude a été initiée pour déterminer les types d'HPV associés aux lésions précancéreuses et cancéreuses du col utérin à Bamako au Mali.

\section{Méthodes :}

II s'agissait d'une étude descriptive prospective qui s'est déroulée du 1er Janvier 2016 au 30 Avril 2017. Au service d'Anatomie et Cytologie Pathologiques (ANAPATH) du CHU du Point-G et au Centre de Recherche Biomoléculaire Pietro Annigoni (CERBA/LABIOGENE) de Ouagadougou (Burkina Faso). Le volet de Ouagadougou a consisté au génotypage des HPV des échantillons recueillis. Les biopsies ont été réalisées sur des cols suspects à l'inspection visuelle après application d'acide acétique à $5 \%$ (IVA) et de soluté de lugol (IVL) des femmes âgées de 20 à 72 ans se présentant dans les différents centres de dépistage. Les fragments de biopsie ont été immédiatement fixés au formol à $10 \%$ ensuite transportés à l'ANAPATH du CHU du Point $G$ où ils ont été inclus dans des blocs de paraffine pour l'analyse histologique.

Au CERBA/LABIOGENE de Ouagadougou, pour le génotypage des HPV à haut risque par PCR à temps réel nous avons utilisé les réactifs suivants : FFPE DNA Purification Kit, pour l'extraction de l'ADN du HPV. PCRRT kit : "HPV génotypes 14 Real-TM Quant » de Sacace Biotechnologies S.r.l, Italie, pour l'amplification de l'ADN. Nous avons inclus tous les cas de LIEHG (CIN2 et CIN3) et cancers invasifs du col utérin diagnostiqués à l'histologie avec étude moléculaire pendant la période d'étude. Au préalable, les femmes ont été soumises à un questionnaire préétabli et leurs consentements ont été obtenus dans les centres qui ont envoyé les pièces de biopsies et le service de I'ANAPATH a donné son avis favorable pour l'étude.

La saisie et l'analyse des données ont été effectuées sur le logiciel SPSS 18.0. Comme test statistique, nous avons utilisé le test exact de FISHER avec un seuil de signification $p=0,05$.

\section{Résultats}

Des cent quatre-vingt-douze (192) femmes ayant bénéficié du test HPV, 83 étaient positives soit $43,2 \%$ (Tableau 1). Les types HPV18 et HPV45 avaient prédominé avec $16,3 \%$ chacun. Parmi les 83 cas de HPV positifs, 64 étaient infectés par un seul type d'HPV soit $77,1 \%$ et 19 par deux ou plusieurs types d'HPV soit
22,9\%. Parmi les infections avec un seul type HPV, HPV16 et HPV18 étaient prédominant avec 18 cas $(\mathbf{2 8}, 1 \%)$ pour chacun. La majorité des femmes avec un HPV positif $(30,9 \%)$ était des multipares et seulement $7,4 \%$ étaient nullipares. Le type histologique le plus représenté était CIN2 soit de $\mathbf{4 2 , 2 \%}$ (Tableau 2). Parmi les cas positifs la tranche d'âge la plus représentée était de $31-45$ ans avec 29 cas soit $34,9 \%$ (Tableau 3). Dans les LIEHG et dans le cancer épidermoïde, les types HPV18 et HPV45 étaient les plus représentés avec un total de 17 cas chacun soit $15,8 \%$. Seulement 3 cas d'HPV (HPV18, HPV45, HPV52) étaient retrouvés dans les adénocarcinomes.

\section{Discussion}

Le HPV à haut risque a été détecté sur 43,22\% (83/192) des lésions cervicales. Steinau et al. en 2011 trouvent $77,6 \%$ (94/121) [5]. Dans notre étude, cette fréquence plus basse pourrait s'expliquer principalement non seulement par l'excès de paraffine qui influence négativement l'extraction de l'ADN mais aussi la manière de conservation des blocs de paraffine. En effet, en comparant deux méthodes de déparaffinage Steinau et al. obtiennent des résultats meilleurs avec la technique de déparaffinage par chauffage, qui a consisté à fondre la paraffine sous la chaleur, que celle utilisant le xylène Steinau et al. [5]. Nous avons trouvé 13 génotypes des HPV à haut risque sur les 14 recherchés, dans les blocs de paraffines. Ce nombre est nettement inférieur à celui trouvé par Nubia Munoz et al. qui trouvent 15 génotypes [2] ; Les génotypes les plus fréquents étaient, par ordre décroissant, HPV18, HPV45, HPV33, HPV31, HPV16 et HPV35. Antérieurement, au Burkina Faso, Djigma et al [6] avaient identifié chez 250 femmes HIV+ les HPV18 (25\%); HPV50'S (25,5\%); HPV30'S (20,8\%); HPV16 $(4,7 \%) ;$ HPV45 (3,7\%). Ce résultat indique une fréquence élevée des HR-HPV autres que les HPV16 et 18. Par contre en France Jean-Luc Prétet et al. trouvent que les génotypes les plus répandus étaient HPV $16(73 \%)$ et HPV 18 (19\%), suivis des HPV 31 (7\%), 33, 68, 45, 52 et 58 (4,1 à 2,3\%) [7]. En Mozambique, on notait une nette prédominance de HPV16 et 18 [8]. Les infections avec un seul type d'HPV étaient majoritaires avec $77,1 \%$ (64/83) et celles avec deux ou plusieurs types d'HPV ont représenté $22,9 \%$ (19/83). Ce résultat est superposable à celui de Nubia Munoz et al. qui trouvent $91,9 \%$ pour les infections avec un seul type de HPV et $8,1 \%$ avec plusieurs types [2].

\section{Conclusion}

Notre étude a montré la présence de 13 génotypes de HPV à haut risque sur les 14 recherchés, les HPV18, HPV45, ont été les génotypes les plus fréquents. Les infections aux HPV, en particulier à ceux appartenant aux génotypes à haut risque oncogène, étaient fortement associées à l'apparition des lésions précancéreuses et cancéreuses du col de l'utérus. La distribution et la fréquence de ces types oncogènes d'HPV diffèrent fortement selon les régions géographiques. II convient de 
connaitre davantage la distribution et la prévalence des types d'HPV à haut risque au sein de la population afin d'adapter les stratégies de vaccination et d'interventions pour lutter contre le cancer du col de l'utérus.

\section{REFERENCES :}

1. Traoré $\mathrm{CB}$, Coulibaly $\mathrm{B}$, Kamaté $\mathrm{B}$, et al. Le cancer à Bamako de 2002 à 2012; données du registre des cancers. Rev. Afr de pathol 2012 6M ; 11(1) : 3-8.

2. Nubia Munoz et al. Epidemiologic Classification of Human Papillomavirus Types Associated with Cervical Cancer. N Engl J Med 2003; 348: 518-527

3. Baldauf J J, Drefus M, Ritter J, Philipe E. An analysis of the factor involved the diagnostic accuracy of colposcopically directed biopsy. Acta obst et gynecol Scand 1997; 76: 468-473.

4. hhtps://www.iccp-portal.org/ressources/les cancersen-afrique-francophone

5. Steinau M, Patel SS, \& Unger ER. Efficient DNA extraction for HPV genotyping in formalin-fixed, paraffin-embedded tissues. J Mol Diagn 2011; 13 : 377-81.

6. Djigma F, Ouedraogo $W$ et al. Prevalence and genotype characterization of human papillomaviruses among HIVseropositive in Ouagadougou, Burkina Faso. Acta Trop 2011 ; 117 : 202-6.

7. Jean-Luc Prétet et al. Distribution du génotype du papillomavirus humain (HPV) dans les cancers invasifs du col utérin en France. Int. J. Cancer 2008; 122: 428-432.

8. Naucler $P$, Flora Mabota da Costa F, Ljungberg $O$; Bugalo A. \& Dillner J. Human papillomavirus genotypes in cervical cancers in Mozambique. J Gen Virol 2004; 85: 2189-90.

Liste des tableaux :

Tableau I : Répartition des patientes selon le type HPV

\begin{tabular}{lcc}
\hline Types HPV & Fréquence & $\%$ \\
\hline HPV18 & 18 & 16,36 \\
HPV45 & 18 & 16,36 \\
HPV33 & 15 & 13,63 \\
HPV31 & 14 & 12,72 \\
HPV16 & 9 & 8,18 \\
HPV35 & 9 & 8,18 \\
HPV52 & 7 & 6,32 \\
HPV39 & 5 & 4,54 \\
HPV56 & 4 & 3,63 \\
HPV58 & 3 & 2,72 \\
HPV59 & 3 & 2,72 \\
HPV66 & 3 & 2,72 \\
HPV51 & 2 & 1,81 \\
Total & 110 & 100 \\
\hline
\end{tabular}

Tableau II : Répartition des patientes selon le test HPV et la tranche d'âge

\begin{tabular}{lccc}
\hline Tranche d'âge & \multicolumn{2}{c}{ Test HPV } & Total \\
\cline { 2 - 3 } & Positif & Négatif & \\
\hline $15-30$ ans & 24 & 23 & 47 \\
$31-45$ ans & 29 & 49 & 78 \\
$46-60$ ans & 18 & 29 & 47 \\
$61-75$ ans & 12 & 7 & 19 \\
$76-90$ ans & 0 & 1 & 1 \\
Total & $\mathbf{8 3}$ & 109 & 192 \\
\hline
\end{tabular}


Tableau III : Répartition des résultats selon le type histologique et le type HPV

\begin{tabular}{lccccc}
\hline & \multicolumn{3}{c}{ Type histologique } & \\
\cline { 2 - 5 } Type & CIN2 & CIN3 & Carcinome & $\begin{array}{c}\text { Adénocarcinome } \\
\text { Épidermoïde }\end{array}$ & Total \\
& & & & & \\
\hline HPV16 & 2 & 2 & 5 & 0 & 9 \\
HPV18 & 9 & 0 & 8 & 1 & 18 \\
HPV31 & 5 & 4 & 5 & 0 & 14 \\
HPV33 & 6 & 4 & 5 & 0 & 15 \\
HPV35 & 3 & 4 & 2 & 0 & 9 \\
HPV39 & 3 & 1 & 1 & 0 & $\mathbf{5}$ \\
HPV45 & 10 & 3 & 4 & 1 & $\mathbf{2}$ \\
HPV51 & 0 & 1 & 1 & 0 & $\mathbf{7}$ \\
HPV52 & 3 & 0 & 3 & 1 & $\mathbf{3}$ \\
HPV56 & 0 & 1 & 3 & 0 & 3 \\
HPV58 & 0 & 0 & 3 & 0 & $\mathbf{3}$ \\
HPV59 & 2 & 1 & 0 & 0 & 110 \\
HPV66 & 2 & 1 & 0 & 0 & \\
Total & 45 & 22 & 40 & 3 & \\
\hline
\end{tabular}

